\title{
Programación de Riego Basado en el Método de Evapotranspiración Para Papaya (Carica papaya) en Florida ${ }^{1}$
}

\author{
Haimanote K. Bayabil, Jonathan H. Crane, Kati W. Migliaccio, Yuncong Li, Fredy Ballen, y \\ Sandra Guzmán²
}

\section{Introducción}

La papaya (Carica papaya) es un importante cultivo frutícola que se cultiva en el sur de Florida con un área estimada de 356 acres (Crane 2018). El condado de MiamiDade representa cerca del $81 \%$ de la producción de papaya en Florida. El valor estimado de la producción de papaya es de $\$ 1.9$ millones basado en un rendimiento promedio de 29,000 libras por acre, una tasa del $85 \%$ de producto empacado versus total producido, y un precio de $\$ 0.40$ por libra. Las prácticas culturales y pautas de manejo de plagas y enfermedades típicas del cultivo de papaya son presentadas en Crane y Mossler (2008). Se probaron tres métodos de programación de riego (programación fija, basada en ET y basada en tensiómetros) en producción de papaya en el sur de Florida. Se encontró que la programación de riego basada en ET conserva el agua de manera efectiva. Este documento se centra en las técnicas de programación de riego basadas en ET para la papaya en las condiciones de Florida.

\section{Importancia del riego de papaya en Florida}

En promedio, Florida recibe de 40 a 60 pulgadas (1000 a $1500 \mathrm{~mm}$ ) de lluvia por año (Zhang et al. 2017). Sin embargo, la distribución espacial y temporal de la lluvia es irregular, lo que resulta en períodos de sequía y humedad excesiva del suelo. Esta discrepancia en la distribución, combinada con suelos arenosos y calcáreos de bueno a excesivo drenaje y con baja capacidad de retención de agua, hace que el riego sea necesario para el óptimo crecimiento y producción de la papaya. Las plantas de papaya estresadas por la sequía dejan caer flores, hojas y frutos jóvenes lo que produce frutos más pequeños con menor contenido de azúcar. El manejo del riego, usando bases científicas, es la clave para garantizar un suministro de agua adecuado para esta planta de rápido crecimiento. Tradicionalmente, la gestión del riego utilizaba programación basada en calendarios de riego. Esta programación resulto no ser eficiente debido a la posibilidad de exceso o déficit de riego, lixiviación de agua, nutrientes y productos químicos por debajo de la zona de las raíces, y la subsecuente falta de disponibilidad de agua

1. Este documento, AE547 (the English version of this document is ET-Based Irrigation Scheduling for Papaya (Carica papaya) in Florida (AE540)), es uno de una serie de publicaciones del Departamento de Ingenieria Agricola y Biologica, Servicio de Extensión Cooperativa de la Florida, Instituto de Alimentos y Ciencias Agrícolas, Universidad de la Florida (UF/IFAS Extension). Fecha de primera publicación: noviembre 2020. Visite nuestro sitio web EDIS en <https://edis.ifas.ufl.edu>.

2. Haimanote K. Bayabil, assistant professor, Department of Agricultural and Biological Engineering, UF/IFAS Tropical Research and Education Center; Jonathan H. Crane, professor, Horticultural Sciences Department, UF/IFAS TREC; Kati W. Migliaccio, chair and professor, Department of Agricultural and Biological Engineering; Yuncong Li, professor, Department of Soil and Water Sciences, UF/IFAS TREC; Fredy Ballen, economic analysis coordinator, UF/IFAS TREC; and Sandra Guzmán, assistant professor, Department of Agricultural and Biological Engineering, UF/IFAS Indian River REC; UF/IFAS Extension, Gainesville, FL 32611.

The Institute of Food and Agricultural Sciences (IFAS) is an Equal Opportunity Institution authorized to provide research, educational information and other services

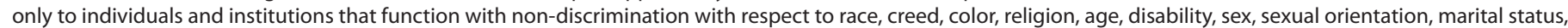

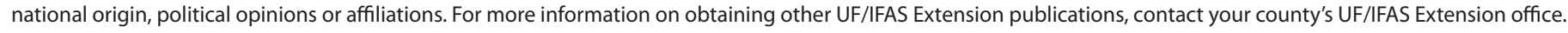
U.S. Department of Agriculture, UF/IFAS Extension Service, University of Florida, IFAS, Florida A \& M University Cooperative Extension Program, and Boards of County Commissioners Cooperating. Nick T. Place, dean for UF/IFAS Extension. 
aprovechable para la planta. Se ha logrado un progreso significativo en el desarrollo de técnicas avanzadas de programación de riego que conservan el agua. Sin embargo, las tasas de adopción de estas nuevas tecnologías por parte de los sectores interesados son bastante bajas, en parte debido a la falta de información sobre los beneficios de estas tecnologías. Este documento proporciona información útil sobre la programación del riego basado en ET para diferentes sectores interesados, incluyendo productores de papaya, agentes de UF/IFAS extensión que trabajan con los agricultores y/o el público en general.

\section{Investigación de riego de papaya en Florida}

Migliaccio et al. (2010) realizaron dos estudios de 2 años para comparar diferentes métodos de programación de riego para la producción de papaya. Los tratamientos incluyeron:

Programación fija: Programa de riego tradicional basado en 1 a 1.5 horas de riego por goteo por día, de 3 a 7 veces por semana, según el clima y la etapa de crecimiento del cultivo. Este método fue usado comúnmente por productores locales en el sur de Florida durante el tiempo en que se diseñó el experimento entre 2006 y 2009.

Programación Basada en ET: Riego basado en la evapotranspiración (ET) utilizando datos históricos de ET provenientes de la estación meteorológica de la Red Meteorológica Automatizada de Florida (FAWN; https://fawn.ifas. ufl.edu/) localizada cerca del área experimental, y valores estimados mensuales del coeficiente de cultivo de papaya $(\mathrm{Kc})$. Los valores de Kc se ajustaron por edad de la planta de la siguiente manera: 1.0, 1.2 y 1.5 para edades entre $0-3$ meses, 4-6 meses, y mayores de 7 meses, respectivamente.

Programación Basada en tensiómetros: Programación del riego basada en el contenido de agua del suelo utilizando valores de tensiómetros de control para iniciar el riego a tres tensiones predefinidas diferentes: $10 \mathrm{kPa}(0.1 \mathrm{bar}), 15$ $\mathrm{kPa}(0.15$ bar) y $25 \mathrm{kPa}(0.25 \mathrm{bar})$. Se instalaron tensiómetros en la zona de raíces, aproximadamente a 1 pie de la base de las plantas de papaya y a una profundidad del suelo de 6.5 pulgadas.

Resultados de estas pruebas de campo: La tasa de aplicación de agua diaria para la programación de riego basada en ET fue entre 34\% y 35\% del tratamiento con programación fija durante los dos años en ambos estudios. De la misma manera, se encontraron ahorros de agua (31\%-36\%) para los tres tratamientos de riego basados en tensiómetros.
Sin embargo, la programación de riego basada en ET puede ser más conveniente y fácil de implementar que el riego basado en tensiómetros. La programación de riego basada en tensiómetros requiere un monitoreo frecuente del tensiómetro para evaluar su precisión y limpieza, mantenimientos periódicos y la reinstalación de tensiómetros con funcionamiento defectuoso. La información, de fácil acceso, usada para la programación de riego por ET está basada en valores históricos o en tiempo cuasi-real y en valores de coeficiente de cultivo $(\mathrm{Kc})$. Con este método, no se necesita equipo de campo.

El crecimiento de la planta de papaya, medido como el diámetro y la altura del tronco, fue mayor con los tratamientos de riego basados en ET y tensiómetros que con el tratamiento de riego con programación fija. Esto sugiere que el programa de manejo de riego con programación fija pudo haber regado en exceso las plantas de papaya, mantenido la zona de raíces demasiado húmeda, y causado un leve estrés por oxígeno y/o la filtración de nutrientes de la zona de la raíz, lo que podrían ser causantes de la reducción en el crecimiento de la planta de papaya.

En general, no hubo una diferencia significativa en el número de frutos y cantidad de frutos producidos por acre alrededor de los tres tratamientos de riego. Sin embargo, el tratamiento con programación fija usó entre un 64\% a 69\% más agua que los tratamientos de riego con ET y tensiómetros, y lo más importante, la producción de fruta por unidad de agua aplicada fue menor para el tratamiento de programación fija comparada con los tratamientos de riego con ET y tensiómetros. El riego con ET y tensiómetros resulto en el uso eficiente del agua con los mismos niveles de producción si se compara con el tradicional método de programación fija de riego.

\section{Implementación de Riego con Programación Basada en ET para Papaya en Florida}

El objetivo de mejorar la programación y la tecnología de riego para la papaya es: (1) ahorrar y usar el agua de manera más eficiente; (2) aumentar el rendimiento y la calidad de los cultivos; (3) potencialmente incrementar el rendimiento del cultivo por unidad de agua utilizada (es decir, más rendimiento por galón de agua aplicado); (4) reducir la lixiviación de agua, nutrientes y productos químicos por debajo de la zona de raíces; y (5) ofrecer métodos de programación de riego fáciles de usar que no aumenten los costos de mano de obra, ni de riego. Además, la implementación de un método de programación de riego 
basado en ET podría ahorrar agua y potencialmente costos de energía sin reducir el rendimiento de los cultivos.

Desde el punto de vista del manejo de cultivo, las ventajas de la programación de riego basada en ET incluyen la no instalación y mantenimiento de sensores de humedad del suelo (tensiómetros), el uso de información de fácil acceso, de ET histórica o en tiempo cuasi-real, y el uso de coeficientes de cultivo $(\mathrm{Kc})$ constantes por mes para calcular la cantidad de agua de riego necesaria. Sin embargo, la disponibilidad de valores confiables de Kc en diferentes etapas del cultivo podría dificultar la programación del riego basado en ET.

La implementación de la programación de riego basada en ET se puede lograr mediante la recopilación de la información como se describe más adelante (parámetros 1 a 4) o mediante la instalación de un controlador de riego inteligente (Dukes et al. 2019). Es importante revisar las capacidades y la disponibilidad de los datos requeridos antes de comprar un controlador de riego inteligente automatizado.

La información necesaria para la implementación de la programación de riego basada en ET es en su mayoría similar para diferentes cultivos, excepto por la información sobre el cultivo en sí. Los pasos generales para la programación de riego basada en ET se describen a continuación en las siguientes publicaciones de EDIS. Algunos de los documentos son parte de una serie sobre programación de riego basada en ET para la agricultura. Esta serie se puede encontrar en https://edis.ifas.ufl.edu/topic_series_ETbased_irrigation_scheduling_for_agriculture (originalmente en inglés). La información también es útil como guía introductoria para controladores de riego inteligentes.

- Kisekka, I., K. W. Migliaccio, M. D. Dukes, B. Schaffer, J. H. Crane, and K. Morgan. 2016. Evapotranspiration-Based Irrigation for Agriculture: Sources of Evapotranspiration Data for Irrigation Scheduling in Florida. AE455. Gainesville: University of Florida Institute of Food and Agricultural Sciences. https://edis.ifas.ufl.edu/ae455

- Kisekka, I., K. W. Migliaccio, M. D. Dukes, J. H. Crane, B. Schaffer, S. M. Guzman, and H. K. Bayabil. 2019. Evapotranspiration-Based Irrigation for Agriculture: Crop Coefficients of Some Commercial Crops in Florida. AE456. Gainesville: University of Florida Institute of Food and Agricultural Sciences. https://edis.ifas.ufl.edu/ae456

- Kisekka, I., K. W. Migliaccio, M. D. Dukes, B. Schaffer, J. H. Crane, H. K. Bayabil, and S. M. Guzman. 2019. Evapotranspiration-Based Irrigation Scheduling for
Agriculture. AE457. Gainesville: University of Florida Institute of Food and Agricultural Sciences. https://edis. ifas.ufl.edu/ae457

- Kisekka, I., K. W. Migliaccio, M. D. Dukes, J. H. Crane, B. Schaffer, H. K. Bayabil, and S. M. Guzman. 2019. Evapotranspiration-Based Irrigation for Agriculture: Implementing Evapotranspiration-Based Irrigation Scheduling for Agriculture. AE458. Gainesville: University of Florida Institute of Food and Agricultural Sciences. https://edis.ifas.ufl.edu/ae458

- Migliaccio, K. W., and Y. C. Li. 2018. Irrigation Scheduling for Tropical Fruit Groves in South Florida. TR001. Gainesville: University of Florida Institute of Food and Agricultural Sciences. https://edis.ifas.ufl.edu/tr001

- Dukes, M. D., M. L. Shedd, and S. L. Davis. 2019. Smart Irrigation Controllers: Operation of EvapotranspirationBased Controllers. AE446. Gainesville: University of Florida Institute of Food and Agricultural Sciences. https://edis.ifas.ufl.edu/ae446

Los parámetros requeridos para la programación de riego basada en ET para papaya se describen a continuación:

\section{Parámetro 1}

Consulte las fuentes de datos de evapotranspiración de referencia estándar (ETo) para la programación del riego (consulte https://edis.ifas.ufl.edu/ae455 para obtener más detalles). ETo está disponible para períodos actuales o históricos y ambos se pueden utilizar para calcular la demanda de agua de los cultivos.

\section{Obtención de valores ET recientes del Sitio Web de FAWN}

- Ingrese a http://fawn.ifas.ufl.edu/.

- Haga clic en Herramientas (tools) en el menú superior.

- Haga clic en Irrigation (riego) - Evapotranspiration (ET) (evapotranspiración) en el menú riego.

- En la parte superior se puede seleccionar la estación meteorológica de FAWN más cercana a su sitio de interés.

- Aparecerá una tabla con la ETo diaria de los últimos 7 días calendario y la ETo promedio de 7 días para cada uno de los sitios de la estación meteorológica FAWN. También está disponible un gráfico con la ETo de los últimos 14 días para sitios FAWN seleccionados. La ET promedio de 7 días se puede usar junto con los valores del coeficiente de cultivo $(\mathrm{Kc})$ para calcular el uso reciente de agua del cultivo para las necesidades de riego de la semana siguiente. 


\section{Valores ET históricos de referencia (ETo)}

Estos datos también se pueden obtener de FAWN haciendo clic en la pestaña del menú Acceso a datos (DATA ACCESS) y seleccionando Generador de informes (REPORT GENERATOR). Por ejemplo, los datos mensuales correspondientes a 10 años pueden descargarse y compilarse para producir un valor promedio de ET de 10 años por mes para Homestead, Florida (Tabla 1).

\section{Parámetro 2}

Obtenga valores estimados del coeficiente de cultivo de papaya $(\mathrm{Kc})$.

\section{Parámetro 3}

Determine la capacidad de retención de agua de los suelos donde se planta la papaya. Se pueden utilizar datos publicados. La información publicada está disponible en varias fuentes, incluida la Encuesta Nacional Cooperativa de Suelos del USDA (http://websoilsurvey.nrcs.usda.gov/ app) y en Migliaccio y Li (2018) (https://edis.ifas.ufl.edu/ tr001 en inglés).

\section{Parámetro 4}

Para la programación de riego basada en ET, calcule la tasa de evapotranspiración mensual real (ETc) multiplicando ETo por los valores del coeficiente de cultivo (Kc) apropiados para la edad de la planta en el campo (Tabla 4).

Luego, calcule la cantidad de riego (I) utilizando los datos de lluvia locales o in-situ y los valores de ETc para la papaya. Por ejemplo, si la lluvia es de 0.1 pulg/día en julio, la cantidad de riego necesaria para las plantas de papaya de 7 meses o más durante el mes de julio se calcula como: $\mathrm{I}=$ ETc $-\mathrm{R}$ (precipitación $)=0.28$ pulg / día -0.1 pulg / día $=$ $0.18 \mathrm{pulg} /$ día.

\section{Parámetro 5}

Determine o utilice las tasas de suministro de riego públicas para el sistema de riego en uso (tasas de emisor). Una cama de suelo con un ancho de 3 pies, 6 pulgadas de profundidad y longitud variable se usan comúnmente en la producción de papaya en el sur de Florida. Por lo general, se usan dos líneas de cinta de goteo o tubo por fila de papaya y, según el tipo comprado, tienen emisores cada seis, ocho o más pulgadas a lo largo de las líneas. Vea la tasa de descarga del fabricante por pie de longitud de tubo, o ejecute el sistema de riego y recoja el agua emitida por una longitud de tubo de 12 pulgadas durante un período de tiempo conocido para calcular la cantidad de agua emitida por pie por minuto. Esta información también es necesaria para configurar un controlador de riego inteligente.

\section{Pasos para programar el riego basado en ET}

Los pasos necesarios para desarrollar una programación de riego basada en ET para la papaya se resumen con ejemplos en la Tabla 5. Se utilizó un 50\% de déficit hídrico (valor comúnmente utilizado) para calcular los requisitos de riego en la Tabla 5.

\section{Resumen}

Los productores comerciales de papaya en Florida tienen varias opciones para mejorar la eficiencia de sus sistemas de riego sin sacrificar el rendimiento y la calidad de los cultivos. Estos incluyen la implementación de una programación de riego basada en ET calculada y administrada manualmente o el uso de un controlador de riego inteligente con opciones de entrada adecuadas para administrar con precisión la programación de riego. La implementación efectiva de un sistema de programación de riego basado en ET conservaría el agua, lo que a su vez reduce el potencial de lixiviación de nutrientes y químicos más allá de la zona de la raíz hacia el acuífero.

\section{Bibliografía}

Carr, M. K. V. 2014. “The Water Relations and Irrigation Requirements of Papaya (Carica papaya L.): A Review." Experimental Agriculture 50 (2): 270-283.

Crane, J. H. 2018. "Tropical Fruit Production in Florida, Trials, Tribulations and Opportunities." Proc. Fla. State Hort. Soc. 131: ix-xii.

Crane, J., and M. Mossler. 2008. "Crop Profile for Papaya in Florida." Accessed on March 3, 2020. https://ipmdata. ipmcenters.org/documents/cropprofiles/FLPapaya2008.pdf

Dukes, M. D., M. L. Shedd, and S. L. Davis. 2019. Smart Irrigation Controllers: Operation of Evapotranspiration-Based Controllers 4. AE446. Gainesville: University of Florida Institute of Food and Agricultural Sciences. https://edis.ifas. ufl.edu/ae446

Evans, E. A., F. H. Ballen, and J. H. Crane. 2012a. An Overview of US Papaya Production, Trade, and Consumption. FE914. Gainesville: University of Florida Institute of Food and Agricultural Sciences. https://edis.ifas.ufl.edu/ fe914 
Evans, E. A., F. H. Ballen, and J. H. Crane. 2012b. 2012 Cost

Estimates of Establishing and Producing Papaya (Carica

papaya) in South Florida. FE918. Gainesville: University of

Florida Institute of Food and Agricultural Sciences. https://

edis.ifas.ufl.edu/fe918

López, T., R. Martínez, O. Puig, P. Paredes, L. S. Pereira, Y. Chaterlan, and G. Hernández. 2010. "Estimation of the Papaya Crop Coefficients for Improving Irrigation Water Management in South of Havana." In VI International Symposium on Banana: XXVIII International Horticultural Congress on Science and Horticulture for People. 179-186. https://www.actahort.org/members/ showpdf? session $=22259$

Migliaccio, K. W., B. Schaffer, J. H. Crane, and F. S. Davies. 2010. "Plant Response to Evapotranspiration and Soil Water Sensor Irrigation Scheduling Methods for Papaya Production in South Florida." Agricultural Water Management 97 (10): 1452-1460.

Posse, R. P., S. Bernardo, E. F. D. Sousa, and R. D. Gottardo. 2008. "Evapotranspiration and Crop Coefficient of Papaya." Engenharia Agrícola 28 (4): 681-690. http://www.scielo.br/scielo. php?pid=S0100-69162008000400008\&script=sci_abstract

Zhang, M., Y. G. Her, K. W. Migliaccio, and C. Fraisse. 2017. Florida Rainfall Data Sources and Types. AE517. Gainesville: University of Florida Institute of Food and Agricultural Sciences. https://edis.ifas.ufl.edu/ae517 
Tabla 1. Promedio mensual de valores de ETo (en pies/día) basados en 10 años de observaciones históricas mensuales en la estación meteorológica localizada en el UF/IFAS Tropical Research and Education Center, Homestead.

\begin{tabular}{|c|c|c|c|c|c|c|c|c|c|c|c|c|}
\hline \multirow[t]{2}{*}{ Año } & \multicolumn{12}{|c|}{ Mes } \\
\hline & ene & feb & mar & $a b r$ & may & jun & jul & ago & sep & oct & nov & dic \\
\hline 2009 & 0.12 & 0.14 & 0.17 & 0.22 & 0.19 & 0.17 & 0.18 & 0.19 & 0.14 & 0.15 & 0.14 & 0.10 \\
\hline 2010 & 0.10 & 0.11 & 0.12 & 0.17 & 0.20 & 0.21 & 0.20 & 0.18 & 0.17 & 0.17 & 0.13 & 0.12 \\
\hline 2011 & 0.11 & 0.14 & 0.18 & 0.21 & 0.22 & 0.20 & 0.18 & 0.16 & 0.16 & 0.13 & 0.13 & 0.12 \\
\hline 2012 & 0.13 & 0.13 & 0.18 & 0.18 & 0.18 & 0.19 & 0.17 & 0.18 & 0.16 & 0.16 & 0.13 & 0.10 \\
\hline 2013 & 0.11 & 0.13 & 0.16 & 0.17 & 0.18 & 0.17 & 0.16 & 0.17 & 0.14 & 0.14 & 0.12 & 0.09 \\
\hline 2014 & 0.09 & 0.14 & 0.17 & 0.20 & 0.22 & 0.17 & 0.17 & 0.19 & 0.15 & 0.15 & 0.12 & 0.10 \\
\hline 2015 & 0.11 & 0.13 & 0.17 & 0.19 & 0.22 & 0.22 & 0.19 & 0.17 & 0.15 & 0.15 & 0.12 & 0.09 \\
\hline 2016 & 0.09 & 0.13 & 0.17 & 0.20 & 0.20 & 0.19 & 0.19 & 0.18 & 0.15 & 0.32 & 0.21 & 0.13 \\
\hline 2017 & 0.15 & 0.17 & 0.22 & 0.23 & 0.24 & 0.18 & 0.20 & 0.20 & 0.18 & 0.16 & 0.13 & 0.13 \\
\hline 2018 & 0.14 & 0.16 & 0.21 & 0.23 & 0.18 & 0.20 & 0.21 & 0.20 & 0.18 & 0.19 & 0.14 & 0.14 \\
\hline Promedio & 0.12 & 0.14 & 0.18 & 0.20 & 0.20 & 0.19 & 0.19 & 0.18 & 0.16 & 0.17 & 0.14 & 0.11 \\
\hline
\end{tabular}

Tabla 2. Coeficientes de cultivo estimados para papaya Kc en el sur de la Florida basados en Migliaccio et al. (2010).

\begin{tabular}{|l|c|l|}
\hline Edad de las plantas en campo & Valores Kc & \multicolumn{1}{c|}{ Comentarios } \\
\hline $0-3$ meses & 1.0 & Recién plantadas y plantas jóvenes. \\
\hline $4-6$ meses & 1.2 & $\begin{array}{l}\text { Las plantas empiezan a florecer y a establecer el fruto; periodo de } \\
\text { desarrollo del fruto. }\end{array}$ \\
\hline 7 meses o más & 1.5 & $\begin{array}{l}\text { Las plantas maduras continúan con la fase de florecimiento y desarrollo } \\
\text { del fruto. }\end{array}$ \\
\hline
\end{tabular}

Tabla 3. Capacidad de retención de agua del suelo (pulgadas de agua por pie de profundidad en el suelo) para diferentes tipos de suelo en el sur de la Florida con base en Migliaccio y Li (2018).

\begin{tabular}{|l|c|c|}
\multicolumn{1}{|c|}{ Tipo de suelo } & \multicolumn{1}{|c|}{$\begin{array}{c}\text { Rango } \\
\text { (pulg/pie) }\end{array}$} & $\begin{array}{c}\text { Promedio } \\
\text { (pulg/pie) }\end{array}$ \\
\hline Limos gravosos (suelo rocoso, condado de Miami-Dade) & $1.0-1.4$ & 1.2 \\
\hline $\begin{array}{l}\text { Margas (suelo calcáreo-arcilloso, zonas de baja altitud en el condado } \\
\text { de Miami-Dade) }\end{array}$ & $1.2-2.4$ & 1.8 \\
\hline Turbas y lodos (al sur del Lago Okeechobee) & & 2.5 \\
\hline Arenas y arenas finas & $2.0-3.0$ & 0.70 \\
\hline
\end{tabular}


Tabla 4. Tasas de Evapotranspiración mensual (ETc) basadas en valores históricos de evapotranspiración potencial (ETo) y valores promedio del coeficiente de cultivo (Kc). (Los valores de Kc fueron obtenidos a través de comunicación personal, Crane 2019).

\begin{tabular}{|c|c|c|c|c|}
\hline \multirow[t]{3}{*}{ Mes } & \multirow[t]{3}{*}{$\begin{array}{l}\text { ET } \text { promedio } \\
\text { (pulg/día) }\end{array}$} & \multicolumn{3}{|c|}{$\begin{array}{l}\text { Edad de las plantas (meses) } \\
\text { ( } \mathrm{K}_{c}=\text { coeficiente de cultivo) }\end{array}$} \\
\hline & & $\begin{array}{c}0-3 \\
\left(K_{c}=1.0\right)\end{array}$ & $\begin{array}{c}4-6 \\
\left(K_{c}=1.2\right)\end{array}$ & $\begin{aligned}>7 \\
\left(K_{c}=1.5\right)\end{aligned}$ \\
\hline & & \multicolumn{3}{|c|}{ ETc (pulg/día) } \\
\hline ene & 0.12 & 0.12 & 0.14 & 0.17 \\
\hline feb & 0.14 & 0.14 & 0.16 & 0.21 \\
\hline mar & 0.18 & 0.18 & 0.21 & 0.26 \\
\hline$a b r$ & 0.20 & 0.20 & 0.24 & 0.30 \\
\hline may & 0.20 & 0.20 & 0.24 & 0.30 \\
\hline jun & 0.19 & 0.19 & 0.23 & 0.28 \\
\hline jul & 0.19 & 0.19 & 0.22 & 0.28 \\
\hline ago & 0.18 & 0.18 & 0.22 & 0.27 \\
\hline sep & 0.16 & 0.16 & 0.19 & 0.24 \\
\hline oct & 0.17 & 0.17 & 0.21 & 0.26 \\
\hline nov & 0.14 & 0.14 & 0.17 & 0.21 \\
\hline dic & 0.11 & 0.11 & 0.14 & 0.17 \\
\hline
\end{tabular}

Tabla 5. Resumen de los pasos para la implementación de un sistema de riego con base en ET.

\begin{tabular}{|c|c|c|c|}
\hline Paso & Fuente de información & Ejemplo/respuesta & Comentarios \\
\hline 1. Seleccione la ETo correspondiente. & Tabla 1 (julio). & $0.17 \mathrm{pulg} / \mathrm{dí} a$ & Valores mensuales para Homestead. \\
\hline $\begin{array}{l}\text { 2. Seleccioneel adecuado Kc con base } \\
\text { en la edad de la planta. }\end{array}$ & $\begin{array}{l}\text { Tabla } 2 \text { (plantas mayores a } 7 \\
\text { meses). }\end{array}$ & $1.5 \mathrm{pulg} / \mathrm{dí} a$ & $\begin{array}{l}\text { Valor de Kc estimado para plantas } \\
\text { desarrolladas. }\end{array}$ \\
\hline $\begin{array}{l}\text { 3. Determine la profundidad activa de } \\
\text { la zona de raíces. }\end{array}$ & Medida en campo. & $6 \mathrm{pul}$ (0.5 pies) & $\begin{array}{l}\text { Valores típicos de profundidad de } \\
\text { hileras en Homestead. }\end{array}$ \\
\hline $\begin{array}{l}\text { 4. Seleccione la capacidad de } \\
\text { retención de humedad del suelo } \\
\text { correspondiente. }\end{array}$ & Tabla 3 (limos gravosos). & $\begin{array}{l}1.2 \text { pulg/pie } \times 0.5 \text { pie } \\
\text { de profundidad }=0.6 \text { - } \\
\text { pulg profundidad de } \\
\text { suelo de la hilera }\end{array}$ & $\begin{array}{l}\text { Tomado de datos previamente } \\
\text { publicados. }\end{array}$ \\
\hline $\begin{array}{l}\text { 5. Determine la tasa de aplicación del } \\
\text { riego. }\end{array}$ & Especificaciones del fabricante. & $0.1 \mathrm{pulg} / \mathrm{hr}$ & Para mayor precisión medir en campo. \\
\hline $\begin{array}{l}\text { 6. Calcule el tiempo necesario para } \\
\text { alcanzar capacidad de campo si el } \\
\text { riego se inicia con un } 50 \% \text { de déficit de } \\
\text { riego. }\end{array}$ & $\begin{array}{l}50 \% \text { déficit de riego } \times 0.6 \text {-pulg } \\
\text { de capacidad de retención de } \\
\text { humedad } \times(0.6-\text { pulg profundidad } \\
\text { de raíces } / 0.1 \mathrm{pulg} / \mathrm{hr}) \text {. }\end{array}$ & $\begin{array}{l}1.8 \mathrm{hr}(1 \mathrm{hr}, 48 \mathrm{~min}) \\
\text { asumir que no se } \\
\text { presentaron lluvias } \\
\text { recientemente. }\end{array}$ & $\begin{array}{l}\text { Asumir no más del } 50 \% \text { de déficit de } \\
\text { riego para iniciar el riego. }\end{array}$ \\
\hline
\end{tabular}

